NASA Technical Memorandum 104418

\title{
Research and Development of Optical Measurement Techniques for Aerospace Propulsion Research-A NASA Lewis Research Center Perspective
}

\section{Daniel J. Lesco}

Lewis Research Center

Cleveland, Ohio

Prepared for the

Second International Conference on Photomechanics and Speckle Metrology sponsored by the Society of Photo-Optical Instrumentation Engineers San Diego, California, July 22-26, 1991

\section{N/SA}




\title{
Research and Development of Optical Measurement Techniques for Aerospace Propulsion Research- a NASA Lewis Research Center Perspective*
}

\author{
Daniel J. Lesco \\ National Aeronautics and Space Administration \\ Lewis Research Center \\ Cleveland, Ohio 44135
}

\section{ABSTRACT}

The applied research effort required to develop new nonintrusive measurement techniques capable of obtaining the data required by aerospace propulsion researchers and of operating in the harsh environments encountered in research and test facilities is discussed and illustrated through several ongoing projects at NASA Lewis Research Center. Factors including length of development time, funding levels, and collaborative support from fluid-thermal researchers are cited. Progress in developing new instrumentation via a multipath approach, including NASA research, grants, and government-sponsored research through mechanisms like the Small Business Innovative Research program, is also described.

\section{INTRODUCTION}

Today's challenges in aerospace propulsion systems lie in the directions of higher combustion and component temperatures, higher airflow velocities within the expanding envelope of what constitutes the "propulsion system," and lower levels of pollution products. Research in any and all of these areas of advanced propulsion systems requires ever more sophisticated instrumentation. Detailed measurements are needed for design code validation, which is of increasing importance to reduce design time and lower the number of iterative designs tested. The harsh environments encountered in experiments and research facilities also put a demand on the measurement systems. The increasing propulsion system temperatures can sometimes exceed the available sensor material limits. Often the mere intrusion of a physical sensor can adversely affect the needed measurement, as in the cases of flow houndary layer studies and high Mach number shock field mapping.

Therefore, a significant need has developed for optical and other nonintrusive measurement systems with the characteristics of accuracy, reliability, and ruggedness. The optical techniques and promising innovative concepts are not lacking; but there is a large gap between demonstrated laboratory techniques and optical measurement systems capable of operating successfully in the facilities used for propulsion research. Some of the difficult factors encountered include vibration and noise, and windows whose design must balance between passing the required radiation wavelength and protecting (from dirt, hot gases, high pressure gases, etc.) what lies on both sides. Also, data must often be obtained in facilities with limited run times which may range from less than a millisecond to a few seconds of total run or stable condition times. Underestimating the gap between operation in the laboratory and in facilities lies probably more on the side of the optical system developers than on the facility operators. Facilities people see operational problems with the simplest sensors in some of the harsh environments and have unfortunately developed some cynicism when asked to install complex optical systems which must operate reliably on demand and within budget.

From the author's perspective as a NASA manager of optical measurement systems research and as a member of a NASAwide aerospace sensors working group, some examples of ongoing optical instrumentation development and the methods by which it progresses are discussed below. Instrumentation research at NASA Lewis has significant involvement in DOD/NASA programs involving advanced propulsion systems (such as National Aerospace Plane (NASP) and Integrated High Performance Turbine Engine Technology (IHPTET)).

\section{NASA INSTRUMENTATION RESEARCH}

Because of NASA's major role in aerospace propulsion systems research, NASA has an accompanying interest and involvement in instrumentation research. But since instrumentation in itself is not an end-product for NASA's endeavors, the research and development leading to new instrumentation technology tends to be thought of as short term and secondary and tied

\footnotetext{
*Invited paper.
} 
to specific aerospace program needs. As in the case of materials research, however, there are common instrumentation technology - challenges that, if met through long term R\&D, will benefit many.

NASA conducts research ${ }^{1}$ in optical measurement systems for aerospace propulsion-related needs, with significant efforts at Ames Research Center, Langley Research Center, Lewis Research Center, and Marshall Space Flight Center. This work includes some grant support and R\&D contracts with private industry. The efforts include both facility- and flight-specific measurement systems. Besides the main thrusts supported by NASA's research programs, within the past few years there has been substantial additional support from supplementary programs, such as the Small Business Innovative Research Program (SBIR), which appear to be a natural fit for the investigation of the unproven innovative ideas which have been generated in basic discipline research. The SBIR program supports a multiphase approach, with a Phase I "proof-of-concept" step funded at $\$ 50,000$ followed by a Phase II funding for up to $\$ 500,000$ for the successful ventures. A government funded Phase III can follow, but generally it is expected that a commercial venture will carry the product to the marketplace. An illustration from NASA Lewis Research Center of this method of optical measurement system development will be discussed in the next section.

Those engaged in propulsion instrumentation research often feel that there are insufficient funds committed to their research relative to the importance of getting successful data. There are several reasons for this perception. It is often difficult to get the propulsion system researchers to define firm instrumentation needs until it is too late to develop new solutions in time for the experimental program. The development time and funds needed for new measurement technology are comparable to those needed to develop the other new technologies required for an advanced propulsion system, except that the phasing of the instrumentation development must lead so that the data is available to enhance the design as the test program proceeds. Also, the need, in the case of research facility instrumentation, is usually for a few to a few tens of measurement systems-not for thousands of systems - so that the market is not substantial enough for many commercial developers to have a long-range interest.

The span of scientific disciplines required to develop an advanced optical instrumentation system can range from physical chemistry to mechanical and electrical engineering. For imaging or spectrum measurement systems, software development skills are usually needed. There must be close support and collaboration from users such as the fluid-thermal researchers (who are often forced to develop their own advanced instrumentation in many organizations if an instrumentation research group does not exist). Facility operations engineers, traditionally responsible for facility instrumentation, may not have the time and specific knowledge to effectively use new optical techniques. Also, some development time is necessary on-line in the target facility or a comparable facility.

There are many other factors influencing the decision to incorporate advanced optical measurement systems in facilities, including safety and the cost of hardware and software.

\section{OPTICAL MEASUREMENT SYSTEMS R\&D AT LEWIS RESEARCH CENTER}

Since Lewis Research Center is NASA's lead propulsion research center, its Instrumentation and Control Technology Division is responsible for the development of instrumentation technology needed for the research, operation, and control of aerospace propulsion systems. The scope of this instrumentation ranges from inlet flow measurements through combustion diagnostics to nozzle plume characterization. As discussed earlier, the emphasis today is on higher temperature and higher speed propulsion systems and hence the drive towards nonintrusive measurements. We will discuss some examples of ongoing NASA Lewis optical instrumentation research and its application in propulsion research facilities.

Although the NASA Lewis research in optical measurement systems is varied and often program or application-specific, there are general features or characteristics which are long-term goals. One goal is multiparameter measurements, wherein one laserbased system determines several parameters simultaneously. Another is whole-field measurements (two-dimensional and threedimensional), in which parameter maps are obtained simultaneously as opposed to scanned point measurements. Also, fiberoptic-coupled systems are advantageous to avoid the problem of structure-disturbing windows in propulsion systems or facilities.

\subsection{Combustion Diagnostics}

There is a significant national effort in developing laser-based combustion diagnostic systems, which have been used routinely in the laboratory, for application to propulsion and combustion facilities. Inelastic scattering techniques such as Raman spectroscopy, including coherent anti-Stokes Raman spectroscopy (CARS), and laser-induced fluorescence (LIF) have been of particular interest because of the potential to measure temperature and species concentrations with good spatial resolution. But these spectroscopy systems are complex and costly to implement, and typically require an advanced degreed specialist to manage the operation and data interpretation of the systems. 
NASA Lewis is working with Rocketdyne and a subcontractor Physical Sciences Inc. to develop for the National Aerospace Plane program a scramjet combustion diagnostic system designated the Multipoint, Multiparameter Hypersonic Flow Diagnostic System (MPMP). The goal of the system is to measure gas path temperature and both major and minor species concentrations in a scramjet combustor under test at various NASP-related facilities. The system, shown in Fig. 1, consists of a dual planar LIF subsystem (one excimer laser plus a neodymium-YAG laser and two intensified solid-state cameras) together with a point Raman subsystem. The planar LIF subsystem will be used to measure either temperature, by means of ratioing two spectral lines of a species like $\mathrm{OH}$, or concentrations of two species (e.g., $\mathrm{OH}$ and NO) simultaneously. ${ }^{2}$ Most major species, such as water, nitrogen, oxygen, and hydrogen, cannot be easily detected using LIF. The Raman subsystem will be used to measure these species; however, the Raman scattering is too weak to be reliably detected when the laser beam is spread into a sheet, so the Raman spectroscopy will be limited to point measurements.

As part of the system development, a partial prototype system consisting of a Nd:YAG laser and one intensified camera were operated in a scramjet combustor test facility to obtain qualitative maps of $\mathrm{OH}$ for several combustor configurations. The laser was remotely located with the UV laser beam sent through a beam tube to the test rig. Representative data from these tests is shown in Fig. 2. We learned some practical lessons from this initial application in the presence of noise, vibration, water, and heat. The tests established a need for a simple (nonimaging) fluorescence detector for quick laser wavelength tuning and showed the advantage of real time image viewing for operator setup. Reliable and stable laser operation is critical, since initiation of data sequences must be on-demand after long holding periods, together with quick restarts after test aborts. Rapid and safe window replacement and cleaning procedures must be available.

The MPMP system is not expected to remain in a stagnant configuration. As measurement technology matures, changes in lasers or detectors will be incorporated. A velocity measurement capability will be added. To date, the primary contribution of this ambitious effort has been the development of a complex system capable of coordinated, synchronized planar data acquisition and processing in a test world environment.

\section{FLOW DIAGNOSTICS}

\subsection{Rayleigh Scattering Diagnostics}

Rayleigh scattering is an elastic process wherein the internal energy of the scattering molecules is not changed by the incident photons. Therefore, it is commonly considered that the scattered light is at the same wavelength as the incident radiation, and that the amount of this scattered light received at a detector can be measured to determine the density of the gas causing the scattering. Ongoing research at NASA Lewis is exploring the less used technique of measuring the spectrum of the Rayleigh scattered light, which contains information about the gas temperature and velocity. In one study, ${ }^{3}$ a spectrally-resolved diagnostic system is being used in hydrogen-oxygen rocket plume research to make multiparameter measurements of plume velocity, temperature, and density. Figure 3 shows the optical configuration of the system. An argon-ion laser supplies about $1 \mathrm{~W}$ of single axial mode radiation needed for the spectral measurements. The scattered light from the plume (the combustion product is predominantly water) is gathered at a near back-scattered angle and directed through a scanning Fabry-Perot interferometer to a cooled photomultiplier. Figure 4 is a photograph of the system installed in the rocket test facility, with the chamber cover drawn back during optical system alignment. Plume data from this experimental set-up is being used to validate plume analytical models of velocity and temperature distributions as a function of radial and axial position.

The spectral width of the scattered light is on the order of $20 \mathrm{GHz}$, which requires a high resolution interferometer. Figure 5 is a simplified interpretation of the method by which the gas parameters are determined from the spectral characteristics. One of the major challenges in applying an elastic scattering technique to a low density medium is in eliminating extraneously scattered light (e.g., from walls and optics) which could overwhelm the weak gas scattered radiation. Particles in the flow would also be very disrupting - even to the velocity measurement unless the particles are moving at the gas velocity and hence imposing the same Doppler shift to the scattered light.

In the rocket plume application, the gas density is low and thus the Rayleigh spectrum is Gaussian. Development of the spectrally-resolved Rayleigh diagnostic technique is also proceeding ${ }^{4}$ for temperature measurements in a high-pressure (1000 psi) furnace, where the spectrum is no longer Gaussian but is composed of a central peak together with two symmetrical side peaks arising from random thermally excited acoustic waves in the gas. Displacement of these wing peaks is related to the temperature of the gas by the speed of sound relationship to the square root of temperature. Figure 6 shows the calculated Rayleigh spectrum of nitrogen at $300 \mathrm{~K}$ and 100 psia. 
In addition to the NASA Lewis Rayleigh diagnostics described, Aerodyne Research developed ${ }^{5}$ for NASA Lewis, under the NASA SBIR program, a dynamic gas temperature measurement system based on the more common detection of total Rayleigh scattered light which is proportional to gas density as it is represented by an average molecular scattering cross section of the gas constituents. (Temperature measurement requires a gas pressure measurement in addition to the gas density provided by the Rayleigh scattering.) This system uses a copper-vapor laser delivering about $20 \mathrm{~W}$ average power at 6000 pulses per second to generate a fairly high level Rayleigh signal per $40 \mathrm{~ns}$ pulse and hence enable dynamic measurements to about a kilohertz. More importantly, the copper-vapor laser generates two lines at 510 and $578 \mathrm{~nm}$. The Rayleigh scattered light is collected by two detectors filtered to receive the two wavelengths. Since the molecular scattering cross section is a function of the wavelength, but the surface-scattered background light is not, surface light can be subtracted from the total signal to give a more accurate measurement of the molecular Rayleigh-scattered light from the probe volume. The system was designed to make combustion temperature measurements in a hydrocarbon-fueled combustor in the presence of soot particles. The high intensity of Rayleigh scattered light from a soot particle causes rejection of the data from laser pulses during which soot is present in the probe volume. The system block diagram is shown in Fig. 7. Fiber-optic cable is used to transmit the laser light from the laser to the probe and the scattered light to the photomultiplier tubes.

This Rayleigh-based diagnostic was proposed by Aerodyne Research as a Phase I SBIR candidate. The result of the Phase I contract was a thorough analytical study of the potential of the technique to provide dynamic temperature measurements in a practical combustor. The Phase II follow-on proposal survived the competition of other successful Phase I contracts to receive a Phase II award. This contract resulted in a deliverable measurement system which has been undergoing subsequent evaluation, refinement, and testing at NASA Lewis. This example further illustrates the significant total effort required to develop a practical optical measurement system. The results have warranted further effort, but the system is not yet ready for facility use.

\subsection{Laser Anemometry}

Laser anemometry is a mature optical measurement technique which has found widespread use in many aerospace applications and facilities. ${ }^{6,7}$ There are two basic classes of laser anemometers used when good spatial resolution is of importance: the fringe-type anemometer often referred to as "laser doppler velocimeter" or LDV and the laser transit anemometer (LTA). The former uses the intersection of two laser beams to generate interference fringes in the probe volume, leading to sinusoidal scattering signals as particles pass through the intersection. The detected frequency is a function of particle velocity and fringe spacing. The latter measures the time-of-flight of a particle as it crosses two parallel laser beams. The time between beam crossings of course depends upon the velocity and the beam separation distance.

A new laser anemometer technique ${ }^{8}$ has been developed jointly by NASA Lewis and Case Western Reserve University and is now being prepared for application in a compressor research facility at NASA Lewis. This laser anemometer configuration is called the Four-Spot Laser Transit Anemometer. It was designed to blend the best features of both the fringe and transit anemometers for research involving internal flows in turbomachinery with small flow passages and complex flow geometries. The beam configuration is shown in Fig. 8. The elliptical shape of the beam cross sections was used to increase the particle trajectory acceptance angle over that of the typical circular beams of an LTA. The use of overlapping orthogonally polarized beams allows much more accurate determination of the particle crossing of the center of the beams. The intensity of the laser beams, even with overlapping, elliptical cross sections, is still higher than that of a fringe configuration. The smaller illuminated sampling area allows measurements to be made closer to the internal walls of a flow passage.

The Four-Spot LTA has been extensively tested in laboratory and instrument development facilities. Representative data showing one of the advantages of the Four-Spot system is illustrated in Fig. 9. It is currently being installed in a small centrifugal compressor facility at NASA Lewis where the flow passages are narrow and highly contoured.

As mentioned above, laser anemometry has been extensively used to study aerospace flows. Since it is a point measurement technique, its use to map complete flow fields can be a lengthy process. And, if the facility is an expensive one to operate, the flow field mapping can also be a costly process. Therefore, whole field and two-dimensional flow velocity measurement techniques are being developed for use in fluids research. Particle Image Velocimetry (PIV) and Particle Displacement Tracking (PDT) are two commonly used names for the general class of two dimensional techniques which use photography, videography, or holography to record the position of particles entrained in a flow. Light sheet illumination, either from lasers or flash lamps, is used to define a plane in the flow field. Some of these techniques can involve long data processing times and the delays inherent in any photography-based technique. Research on a video-based method, with rapid data processing on an 386-class PC, is showing significant progress at NASA Lewis. ${ }^{9}$ 
This effort in PIV is an example of optical instrumentation discipline research which was extensively developed first - for use for a space application (a NASA microgravity fluids experiment) and is now being developed for higher velocity aerospace propulsion applications. This is also an excellent illustration of optical technique development which started at the university research level, is being utilized in an on-going project, and is now available to the technical community for use and commercial development.

The Particle Displacement Tracking technique is summarized in Fig. 10. A laser light sheet illuminates particles seeded into the flow. A CCD camera together with a PC frame grabber board is used to record a sequence of video frames. The number of frames recorded (usually five frames) and the time spacing of frames depends upon the expected particle velocity magnitudes and dynamic ranges. The particle time history is derived from the frame sequence and the particle position at each time is coded on one frame for further processing to extract the velocity vectors. The velocity direction is unambiguously determined. An efficient particle tracking algorithm is used to determine the two-dimensional velocity vector maps. The extensive menu-driven software is available through the NASA Cosmic System. ${ }^{10}$ Figure 11 illustrates the two-dimensional velocity vector maps obtained in the microgravity experiment. The data obtained from the random particle positions has been interpolated to generate this more readily visualized map.

The extension of this PIV development to higher aerospace velocities involves synchronization of the video framing and pulsed particle illumination. The design trade-offs involving seeding size (detected signal signal-to-noise ratio versus flow lag) are exacerbated by the additional imaging requirements for the accurate positioning of the particles in each video frame. Video imaging of $2-\mu \mathrm{m}$ seed particles has been achieved for flow velocities of $100 \mathrm{~m}$ per second.

\section{CONCLUDING REMARKS}

The development of new optical instrumentation systems for aerospace research should not continue to be overlooked as to its importance or to the length of time to achieve "routine" operation. Therefore both research and commercial groups must take advantage of all opportunities to supplement their funding and increase their chances of success, such as through small business programs and collaborative efforts. The fluid-thermal researchers must work hand-in-hand with instrumentation researchers and developers to get the systems that will obtain the data they need and still be compatible with the experimental constraints of time, cost, and physical interactions. A duplication of effort is often perceived in instrumentation development even where it is not warranted (e.g., a laser-induced-fluorescence-based system developed for temperature measurements in a highvelocity, high-altitude wind tunnel has different challenges from that of a LIF system developed for temperature and species measurements in a high temperature combustor operating at $10 \mathrm{~atm}$ pressure).

There is ongoing a large quantity of excellent work in optical instrumentation development, in spite of the obstacles. Many of these systems are now being applied in propulsion research facilities. Papers and conference presentations about these optical instrumentation applications are to be encouraged as strongly as papers about the optical techniques themselves. It is a good time to learn from each other and to de'-emphasize competitive concerns, so that the aerospace research community will more fully accept the advantages of optical measurement systems.

\section{REFERENCES}

1. B.A. Conway, "NASA Programs in Advanced Sensors and Measurement Technology for Aeronautical Applications," in 17th International Council of the Aeronautical Sciences Congress, Vol. 1, AIAA, New York, 242-248 (1990).

2. M. Allen, S. Davis, and K. Donohue, "Planar Measurements of Instantaneous Species and Temperature Distributions in Reacting Flows: A Novel Approach to Ground Testing Instrumentation," AIAA Paper 90-2383 (1990).

3. R.G. Seasholtz, F.J. Zupanc, and S.J. Schneider, "Spectrally Resolved Rayleigh Scattering Diagnostic for HydrogenOxygen Rocket Plume Studies," AIAA Paper 91-0462 (1991).

4. R.G. Seasholtz, "Rayleigh-Brillouin Scattering for High-Pressure Gas Temperature Measurements," in Structural Integrity and Durability of Reusable Space Propulsion Systems, NASA CP-10064, 21-28 (1991).

5. K. Annen, and R. Joklik, "The Development of a Rayleigh Scattering Diagnostic for Density and Temperature Measurements," NASA Contract NAS3-24613 (1987).

6. R.G. Seasholtz, "Application of Laser Anemometry in Turbine Engine Research," NASA TM-83513 (1983).

7. D.C. Rabe, and C.L. Dancey, "Comparison of Laser Transit and Laser Doppler Anemometer Measurements in Fundamental Flows," AIAA Paper 86-1650, (1986).

8. M.P. Wernet, and R.V. Edwards, "Implementation of a New Type of Time-of-Flight Laser Anemometer," Appl. Opt. (5), 644-648 (1986). 
9. M.P. Wernet, "Particle Displacement Tracking for PIV," NASA TM-103288 (1990).

10. M.P. Wernet, "Software Manual for Operating Particle Displacement Tracking Data Acquisition and Reduction System," NASA TM-103720 (1991).

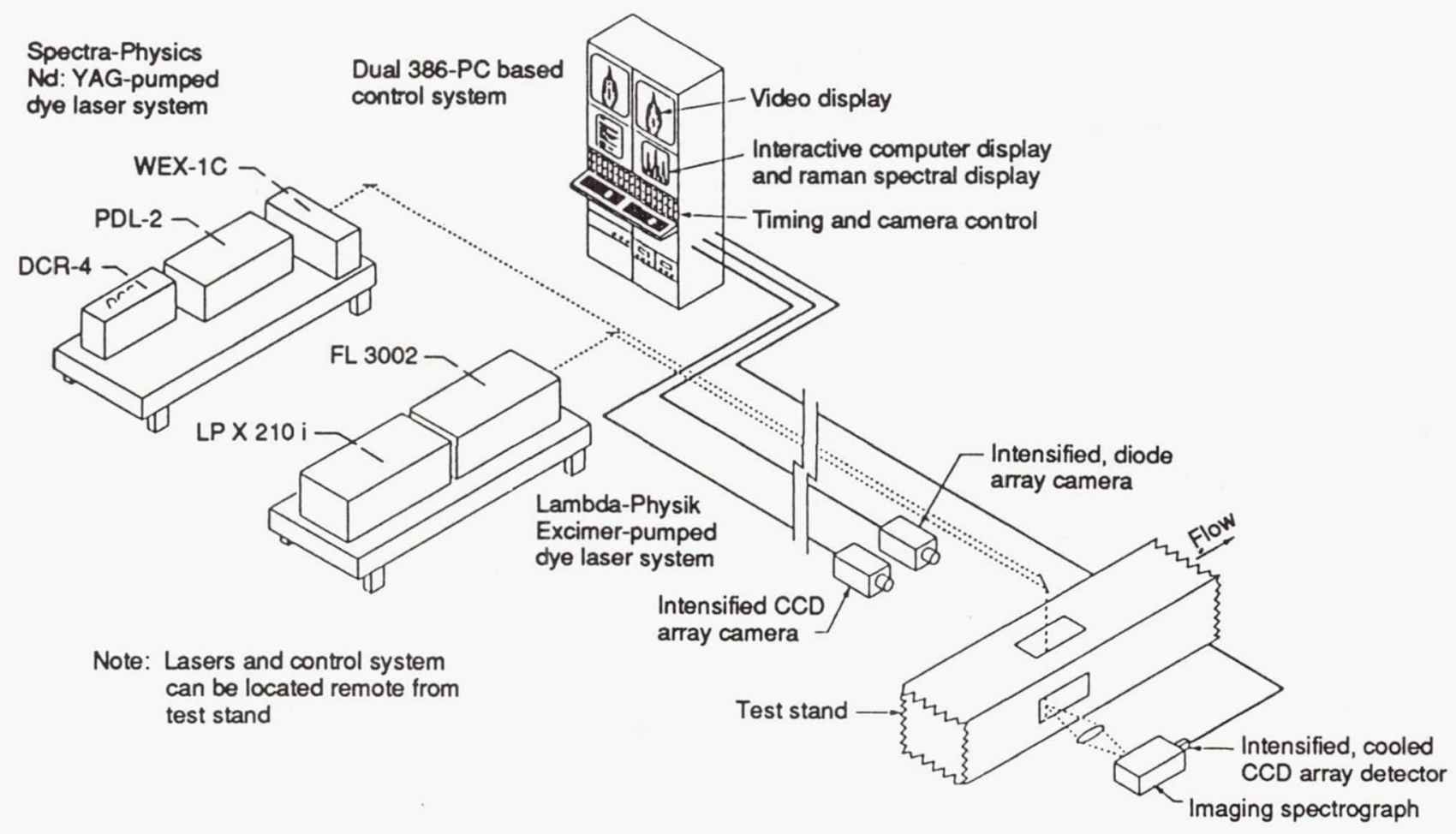

Figure 1. - Multipoint, Multiparameter Diagnostic System for scramjet combustor research.

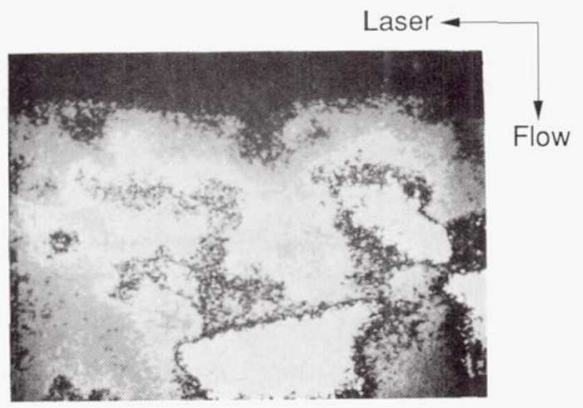

(a) Short combustor.

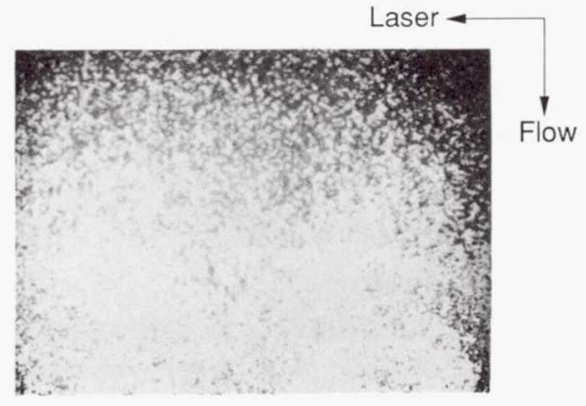

(b) Long combustor.

Figure 2. - Planar Laser-Induced-Fluorescence measurements of $\mathrm{OH}$ concentration in a scramjet combustor, for a) short configuration combustor, b) long configuration. $\mathrm{OH}$ maps indicate more uniform fuel mixing and combustion in long configuration. 


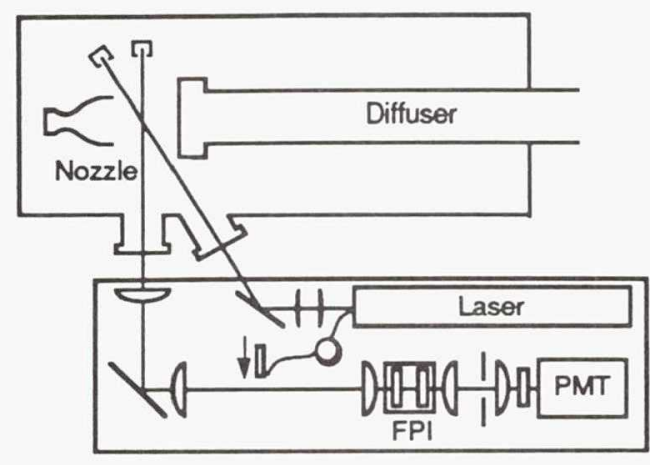

Figure 3. - Optical configuration of Spectrally-Resolved Rayleigh Scattering Diagnostic System for hydrogen-oxygen rocket plume characterization.

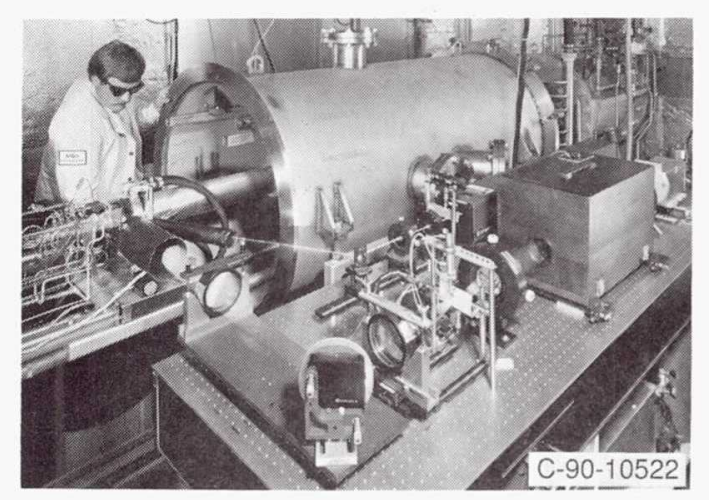

Figure 4. - Photograph of Spectrally-Resolved Rayleigh Scattering Diagnostic System installed in rocket test facility.

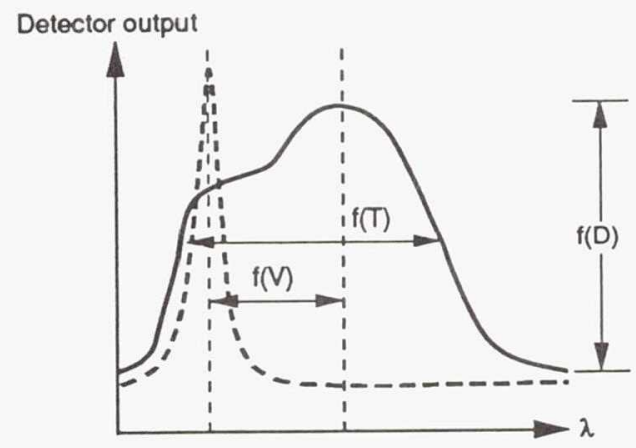

Figure 5. - Simplified Rayleigh spectral pattern for low density gas, for measurement of velocity $(V)$, temperature $(T)$, and density (D).

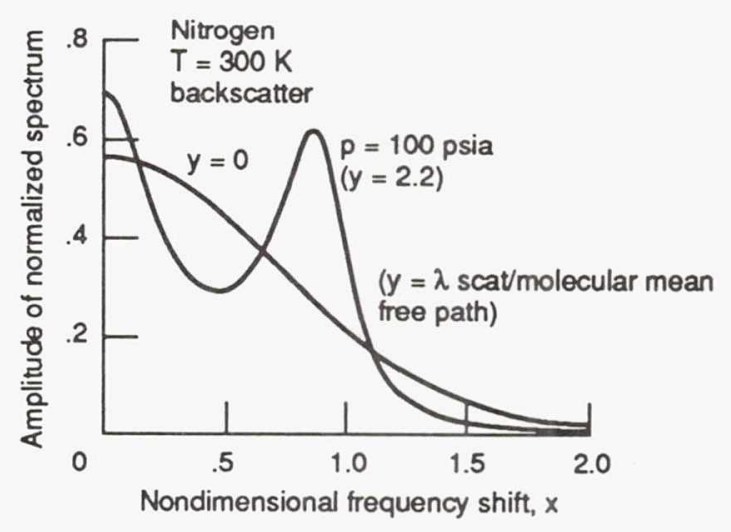

Figure 6. - Calculated Rayleigh spectrum for 100 psia nitrogen at temperature of $300 \mathrm{~K}$.

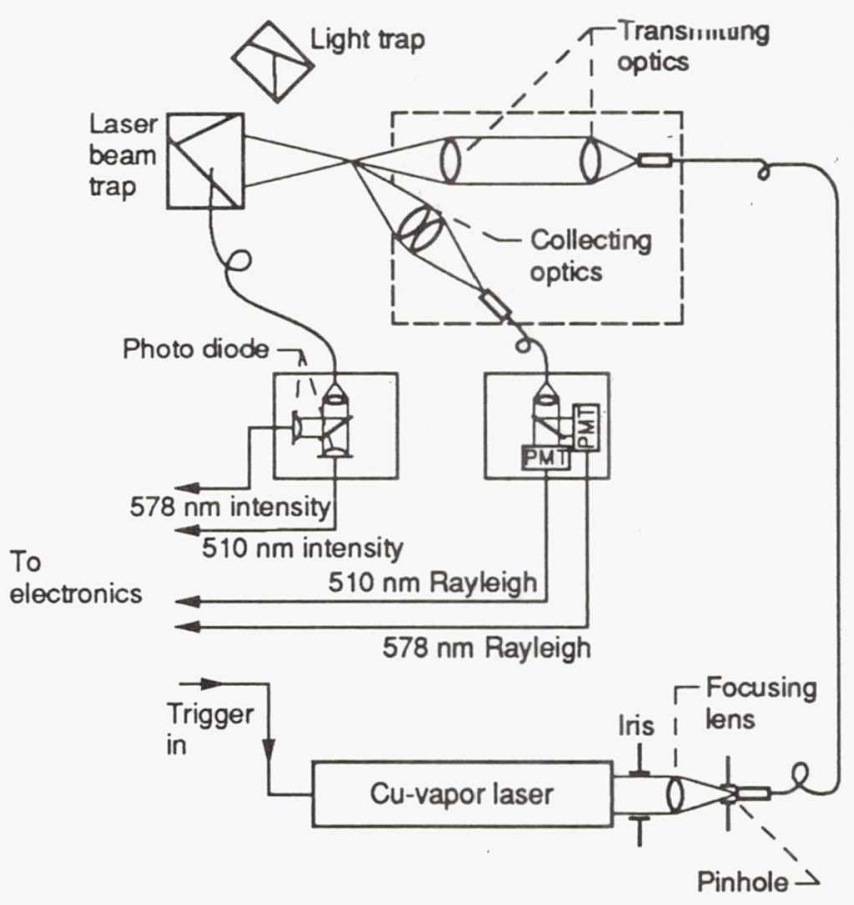

Figure 7. - Optical system block diagram of Rayleigh Scattering Diagnostic System for measuring dynamic gas temperature. 
Z7 Horizontal polarization

$\checkmark$ Vertical polarization

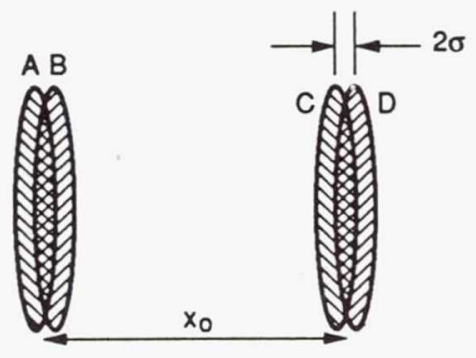

Figure 8. - Four-Spot LTA laser beam cross-section showing overlapping orthogonally polarized spots.

Nominal separation is $100 \mu \mathrm{m}$, spot height is $100 \mu \mathrm{m}$, and spot width is $10 \mu \mathrm{m}$.

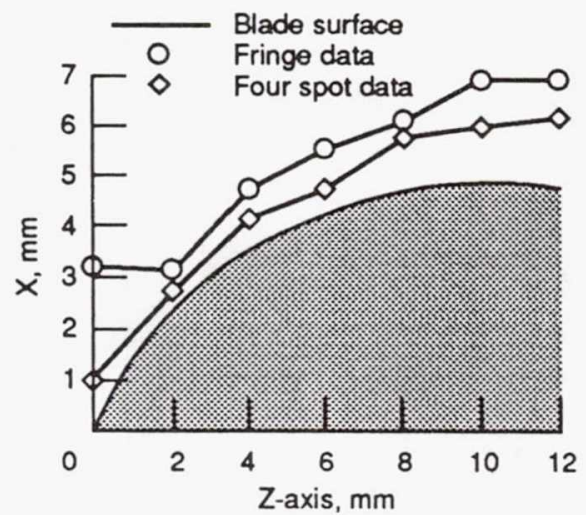

Figure 9. - Comparison of closest proximity-to-surface for measurements using Four-Spot LTA and fringe LA for velocity measurements along a turbine blade surface in a burner rig. 


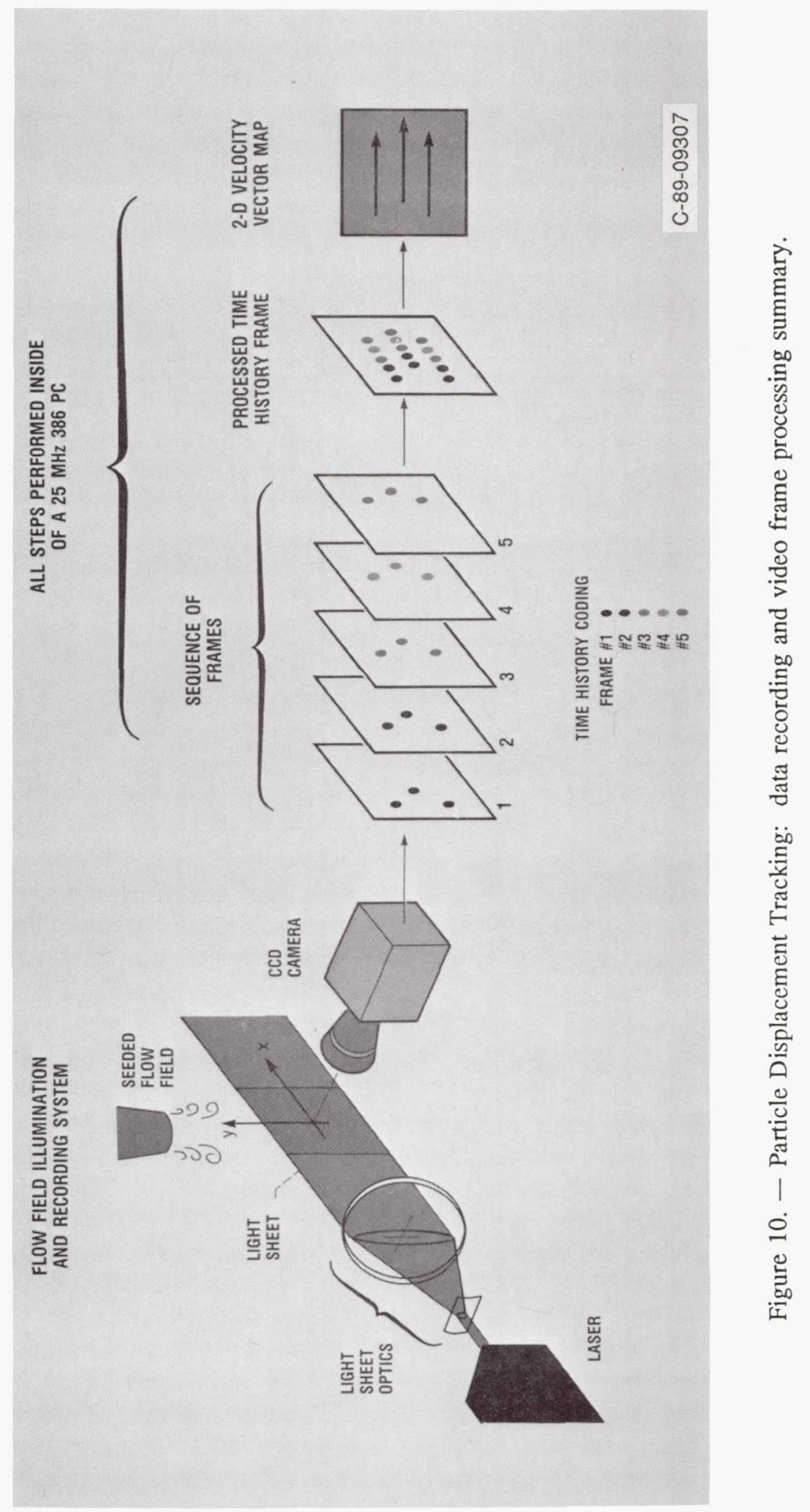




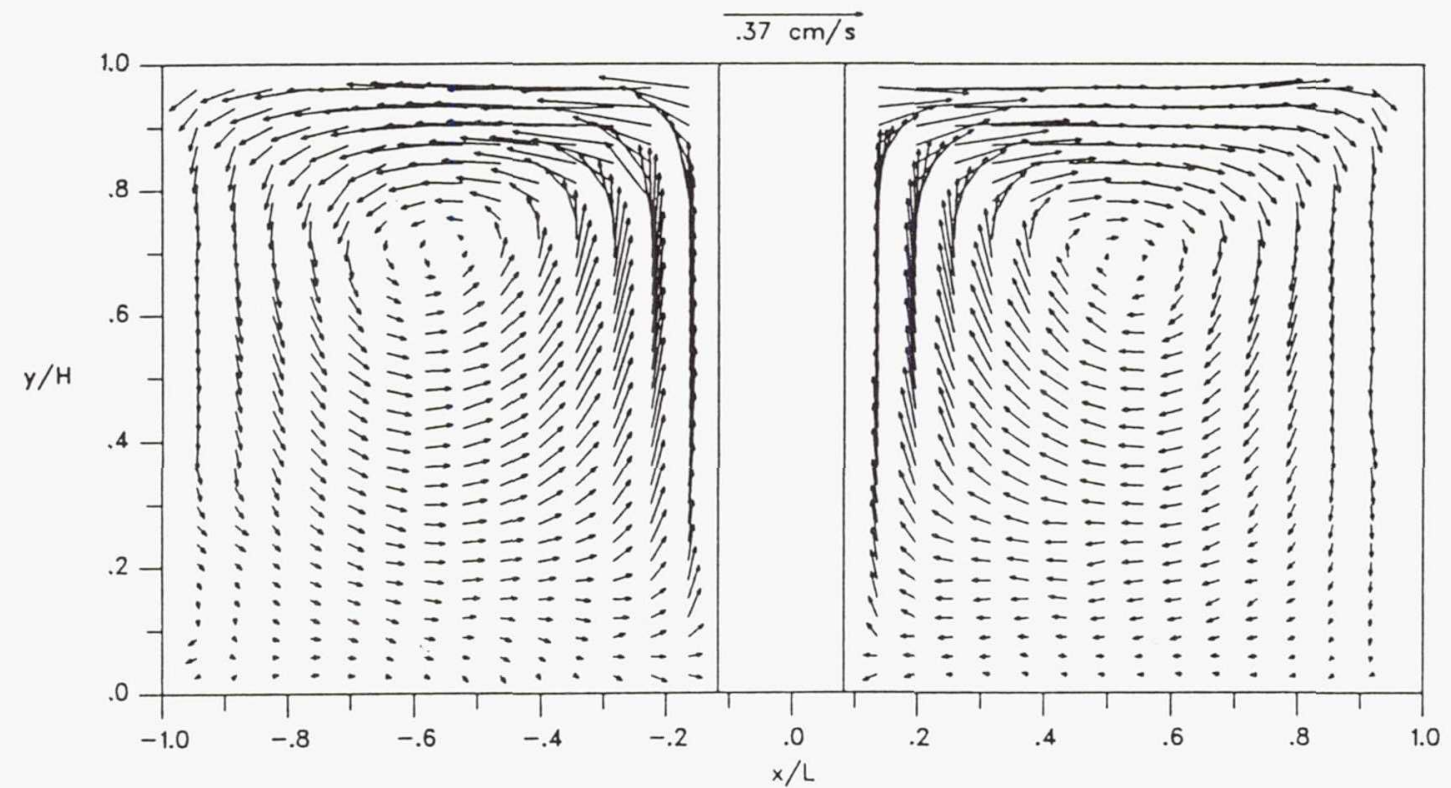

Figure 11. - Interpolated two-dimensional velocity vactor map for microgravity fluids experiment showing symmetrical vortex flow field around central heating zone. 


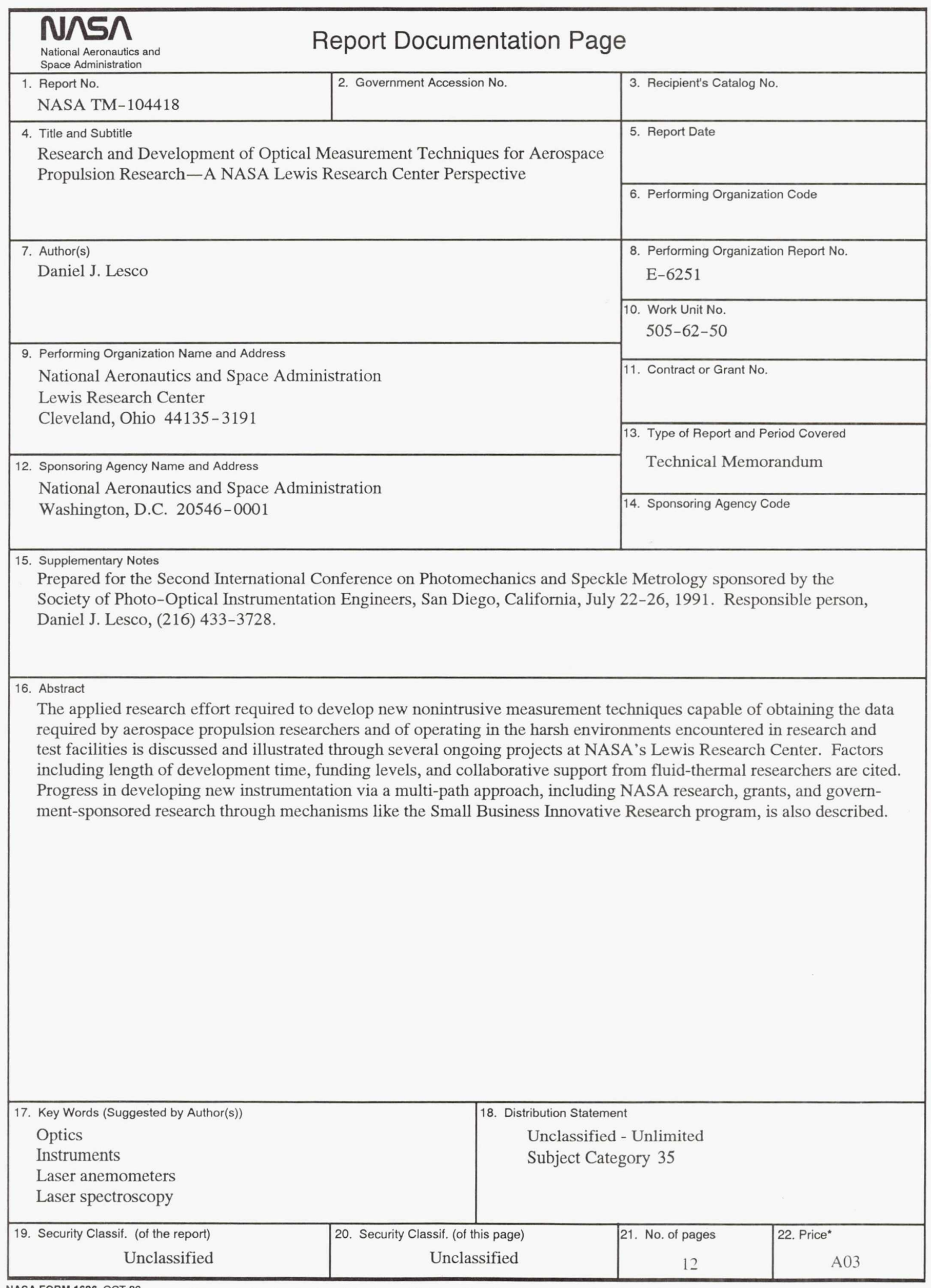


National Aeronautics and Space Administration

Lewis Research Center

Cleveland, Ohio 44135

Official Business

Penalty for Private Use $\$ 300$

Postage and Fees Paid National Aeronautics and Space Administration

NASA 451 